\section{Simple microphotometry}

KP WEST and F WALKER Department of Pathology, Clinical Sciences Building. Leicester Royal Infirmary, PO Box 65, Leicester LE2 7LX, UK

Microphotometry lends itself to extreme approaches varying from the time-honoured, inexpensive, visual appraisal recorded as,+++ , and +++ or the like, to the use of very expensive, purpose-built apparatus recording optical density to the second, or even third, decimal place. The former approach is frequently criticised as subjective; the latter is often derided as esoteric. Though it may appeal to neither the traditionalist nor the purist a simple practical solution to certain microphotometric problems is offered by automatic microscope cameras such as the Leitz Orthomat or the Zeiss Photomicroscope III, which have photomultipliers with masking apertures to facilitate spot exposure measurements.

\section{Procedure}

Ensure that the microscope is optically aligned. Set the film speed indicator towards the lower limit of its range; switch the lamp to its usual intensity for colour photomicrography (about $3700^{\circ} \mathrm{K}$ ), and insert appropriate neutral density filters. The aim of these adjustments is to produce an anticipated exposure time which can be conveniently measured with a stopwatch (usually about 5-10 seconds). Check that the photometer diaphragm is in the correct position for spot readings and that the camera controls are set to trial exposure; alternatively do not put a film in the camera. Select a colour transmission filter appropriate to the chromophore to be measured and place it in the light path of the microscope. Sets of such filters are readily available from microscope manufacturers, but many laboratories still possess filters from long discarded spekkers and similar items of apparatus, and these are equally effective.

Examine the slide to be quantified and centre the desired area in the spot measurement zone of the graticule, selecting the objective so that the spot is filled as completely as possible. This process is facilitated, though not essentially so, by the use of intermediate magnifiers such as the Leitz Vario or Zeiss Optovar. Depress the exposure and stopwatch buttons simultaneously and time the exposure. The Leitz and Zeiss cameras give both visible and

Received for publication 23 July 1979 audible indications when the exposure is complete. Move the slide to an adjacent unstained area and time the exposure here to establish a base-line, or blank, value. The difference between these two readings gives an arbitrary, but reproducible, measurement of the optical density, or transmission, of the desired area.

\section{Application}

Among other things, the procedure has been applied to a study of glomerular polyanion in aminonucleoside nephrosis in rats. Cryostat kidney sections stained with colloidal iron were used to demonstrate the polyanion. The microphotometric results in comparison with subjective appraisal on a - to +++ scale are shown in the Table. The subjective results are comparable with those in a similar aminonucleoside nephrosis study. ${ }^{1}$

Table Glomerular polyanion in aminonucleoside nephrosis measured with a Leitz Orthoplan/Orthomat, $\times 10$ objective, no intermediate magnification, Spekker ORI filter

\begin{tabular}{|c|c|c|}
\hline $\begin{array}{l}\text { Days on } \\
\text { aminonucleoside }\end{array}$ & $\begin{array}{l}\text { Microphotometry } \\
\text { (arbitrary units) }\end{array}$ & $\begin{array}{l}\text { Visual } \\
\text { appraisal }\end{array}$ \\
\hline 0 (control) & $6 \cdot 4$ & $+t$ \\
\hline $\begin{array}{l}0 \text { (control section } \\
\text { treated with } \\
\text { neuraminidase) }\end{array}$ & $1 \cdot 0$ & - \\
\hline $\begin{array}{r}6 \\
8 \\
10\end{array}$ & $\begin{array}{l}3 \cdot 4 \\
2 \cdot 2 \\
2 \cdot 4\end{array}$ & $\begin{array}{l}++ \\
++ \\
+\end{array}$ \\
\hline
\end{tabular}

\section{Discussion}

Comparison of the microphotometric results with the visual appraisal results, particularly the neuraminidase control and the 8- and 10-day treated rats, shows how easily visual appraisal can be subject to bias, thereby conferring a tidy, but debatable simplicity on the observations.

It must be emphasised that a strictly standardised system of tissue preparation is necessary for reliable, quantitative, microscopic examination. Many laboratories are capable of this, but in only a few are the demands for quantitation large enough to warrant the considerable capital expense of a specialised microphotometer. However, many laboratories have automatic microscope cameras, and to them this simplified microphotometric procedure is commended. 
The technical and secretarial assistance of $\mathrm{Mr}$ M. J. L. Rae, Mr T. Pogmore, and Mrs L. Richardson is gratefully acknowledged.

\section{Reference}

1 Michael AF, Blau E, Vernier RL. Glomerular polyanion: alteration in aminonucleoside nephrosis. Lab Invest 1970;23:649-657.

Requests for reprints to: Dr K. P. West, Department of Pathology, Clinical Sciences Building, Leicester Royal Infirmary, Leicester LE2 7LX, UK.

\section{Letter to the Editor}

\section{Immunoperoxidase method for detection of immunoglobulins}

The immunoperoxidase method for the detection of immunoglobulins is a lengthy procedure and often involves a trypsin incubation stage.

In our experience, sections-particularly from skin biopsies-often lift or are lost altogether. Adhesives such as albumin, chrome alum, and amylopectin have been tried without success, and Romac C $33^{1}$ is no longer available. However, Cow Gum, a rubber solution (Li-Lo Ltd, Woking, Surrey, but also available from most stationers) when diluted with a little petroleum ether, smaared onto slides, and allowed to dry is a completely effective adhesive. Sections are then floated onto the prepared slides and incubated at $37^{\circ} \mathrm{C}$ overnight.

The adhesive is clear, does not affect immunoperoxidase reactions, and is sufficiently insoluble in xylene for the sections to be brought to water and finally counterstained in the usual way.

The non-wettable surface of the Cow Gum smear is an advantage in that it limits spreading of the reagents by increasing the angle of contact so that smaller volumes may be used and the risk of the section drying out is reduced.

P G SELLS, M BURTON Department of Parasitology, Liverpool School of Tropical Medicine, Pembroke Place, Liverpool L3 5QA

\footnotetext{
Reference

1 Maxwell A, Ritchie J S D. A comparison of adhesives usable in the cold for the preparation of cryostat sections. Stain Technol $1971 ; 46: 167-169$.
}

\section{Book reviews}

IARC Monographs on the Evaluation of the Carcinogenic Risk of Chemicals to Humans. Volume 19. Some Monomers, Plastics and Synthetic Elastomers, and Acrolein. (Pp. 513; Sw.fr. 60.) Lyon: WHO. 1979.

The industrial production of various monomers and synthetic polymers has grown spectacularly in the last 30 years, but our knowledge of the potential hazards of these ubiquitous and often valuable materials has not developed in a commensurate fashion. Certain acute toxic effects in man have been known for some time in relation to materials such as styrene, acrolein, monomeric vinyl chloride, and the thermodegradation products of polytetrafluoroethylene. Most of the synthetic polymers were regarded as biologically inert until the first experiments were made showing that various plastics, implanted subcutaneously or intraperitoneally into animals, induced local sarcomas. The pathogenesis of such tumours is still obscure and 'solid-state carcinogenesis' remains difficult to reconcile with current genotoxic or epigenetic theories of tumour development. The literature contains isolated case reports of local sarcomas in patients with certain surgical implants but the carcinogenic risks of such materials appear so far to be extremely low. The situation changed abruptly in 1974 when the first human cases of hepatic angiosarcoma were described in workers exposed occupationally to vinyl chloride monomer, and at least 70 examples have now been reported. These are small numbers, but the widescale use of monomeric vinyl chloride and its polymers, and related substances, makes the latest of the IARC monographs particularly timely.

Monomeric vinyl chloride, reviewed by IARC in 1974, is assessed again and its potent carcinogenicity is established beyond doubt. Considerable suspicion now falls on acrylonitrile and, to a lesser extent, on chloroprene and vinyl bromide. The latest monograph follows the same expanded format as the last few volumes in the series, with sections on mutagenicity and teratogenicity and, where appropriate, on intermediate metabolism. This latest volume provides a useful introduction to the clinical and experimental toxicology of a complex and still ill-understood group of substances in very wide daily use.

R. L. CARTER

Experimental Haematology Today. Edited by $\mathrm{S}$. J. Baum and G. D. Ledney. (Pp. $x v i+256$; illustrated; DM 68 , US $\$ 34$.) Berlin: Springer-Verlag. 1978.

This volume is a presentation in book form of papers read at the Sixth Annual Meeting of the International Society for Experimental Haematology held in Basle, Switzerland in 1977. It is not a complete document of the meeting but rather a synthesis of the major contributions. The editors are to be congratulated on achieving a cohesive, well laid-out book within a year of the original meeting.

There are six sections to the book, covering a spectrum from fundamental experimental work to clinical bone marrow transplantation. It includes papers on the abrogation of graft rejection in dogs, and an interesting paper on antitumour effects of syngeneic marrow 